\title{
Jogos digitais educativos em línguas de sinais: uma revisão sistemática da literatura
}

\author{
David Pereira Neto \\ IFSC - Câmpus Palhoça Bilíngue; davidpereiraneto@gmail.com \\ Maria José Baldessar \\ UFSC - Departamento de Jornalismo; mbaldessar@gmail.com
}

\begin{abstract}
Resumo: Numa perspectiva de educação bilíngue para surdos os materiais didáticos devem, preferencialmente, ser desenvolvidos na língua natural dos alunos, a língua de sinais. Os jogos digitais educativos são artefatos multimídia com potencial de aprendizagem discutidos na literatura especializada que permitem o desenvolvimento de materiais didáticos que atendam tal especificidade. Neste artigo será apresentada uma revisão sistemática da literatura que objetiva mapear o estado atual do desenvolvimento de jogos digitais educativos que utilizem línguas de sinais como meio de comunicação. A partir da coleta de publicações nas bases de dados SCOPUS e ERIC são documentados e descritos 24 jogos digitais. A maioria dos jogos apresentados têm como objetivo de aprendizagem a aquisição de linguagem. Jogos de correspondência são a mecânica mais comum entre eles e os avatares tridimensionais são a forma de representação das línguas de sinais mais utilizados.
\end{abstract}

Palavras-chave: jogos digitais; jogos educativos; surdo; língua de sinais; bilíngue.

\section{Digital educational games in sign language: a systematic literature review. Abstract:}

In a bilingual education approach for the deaf, teaching materials should preferably be developed in the students' natural language, sign language. Digital educational games are multimedia artifacts with learning potential discussed in specialized literature that allow the development of teaching materials that meet this requirements. This article presents a systematic literature review that aims to map the current state of digital educational games development that use sign language as communication means. From SCOPUS and ERIC databases research, 24 digital games are documented and described. Most of the presented games has language aquisition as learning objective; matching games are the most common gameplay among them and; three-dimensional avatars are the most used sign language representation way.

Keywords: digital games; educacional games; deaf; sign language; bilingual.

\section{INTRODUÇÃO}

Partindo de uma perspectiva de educação bilíngue de surdos, entende-se que os materiais didáticos devem, preferencialmente, ser desenvolvidos na língua natural dos alunos, em línguas de sinais (LS). Deve-se projetar o material didático com foco em indivíduos que dependem essencialmente de suas experiências sensoriais visuais e das línguas de sinais, pontos nos quais se apoiam a cultura surda e a pedagogia do surdo (MEC, 2014). A "cognição dos surdos se desenvolve de um modo totalmente visual, diferente dos ouvintes que utilizam a audição para se comunicar, para captar explicações, conceitos, significados." (MEC, 2014, p.13) 
Por se tratarem de artefatos multimídias os jogos digitais educativos podem ser desenvolvidos com ênfase nessa pedagogia visual da qual prescinde a educação bilíngue de surdos. Além disso, têm potencial para representar as LS que são essencialmente viso-espaciais (Campelo, 2007). A combinação de imagens, vídeos, animações e outros recursos visuais dispostos em narrativas interativas e desafiadoras é inerente aos jogos digitais (Salen e Zimmerman, 2012).

Os jogos educativos digitais são multimídias interativas que proporcionam situações de construção de conhecimento e que podem ser utilizados para processos de aprendizagem na sala de aula (Jappur, 2014; Busarello, 2016). De acordo com Gee (2003 apud Jappur, 2014, p. 29), os jogos digitais "[...] são adequados para as novas formas de aprendizagem. Possuem contextos poderosos que representam mundos virtuais, e a ação em tais mundos torna possível o desenvolvimento do conhecimento."

Parte de uma pesquisa mais ampla que objetiva o desenvolvimento de jogos digitais educativos em LS, o presente artigo relata o mapeamento de jogos digitais educativos que utilizem LS como forma de comunicação. Para tanto, foi empreendida uma revisão sistemática da literatura com objetivo de encontrar publicações que apresentem o desenvolvimento ou avaliação de tais jogos digitais educativos. A metodologia e etapas da pesquisa são descritas a seguir.

\section{Metodologia}

Esse estudo baseou-se na revisão bibliográfica sistemática (RBS) apresentada por Conforto, Amaral e Silva (2011), a RBS Roadmap. De acordo com os autores, o emprego da revisão sistemática permite que o pesquisador compreenda e dimensione de maneira mais confiável o corpo de conhecimento referente a um determinado assunto. Dessa maneira uma RBS é útil para "[...] ajudar o pesquisador no dimensionamento e compreensão do corpo de conhecimento referente a um determinado assunto, incluindo identificar pesquisas que já foram realizadas, o que falta pesquisar, quais são as lacunas", provendo um "[...] embasamento teórico sólido para o estudo proposto" (idem, p. 4). A RBS está dividida nas fases de Entrada, Processamento e Síntese e Resultados apresentadas a seguir.

\subsection{Fase 1 - Entrada}

Da fase de entrada do RBS Roadmap foram realizadas as seguintes etapas: definição de objetivos; definição de strings de busca; e critérios de inclusão dos documentos.

$\mathrm{O}$ seguinte objetivo de pesquisa foi definido: elencar jogos digitais educativos que utilizem LS apresentados na literatura acadêmica. A partir disso realizou-se a coleta de documentos que indiquem o desenvolvimento ou avaliação de algum jogo digital que utilize alguma forma de representação de LS para surdos em algum momento do jogo.

Para tanto, foram delimitados os seguintes tópicos para a busca: línguas de sinais, jogos e surdo. Com os operadores booleanos e tradução dos termos esses tópicos resultaram no seguinte string de pesquisa: ("sign language" AND game*) OR (deaf* AND game).

Como delimitação ou critérios de inclusão e exclusão dos documentos, foram descartados da pesquisa estudos que: a) apresentem aplicativos restritos à tradução de línguas de sinais; b) descrevam o desenvolvimento de jogos não digitais; c) descrevam o desenvolvimento de jogos digitais que não utilizem línguas de sinais para apresentar informações; d) apresentem somente modelos ou bases teóricas relacionadas a jogos para surdos, usuários de línguas de sinais ou aprendizes de línguas de sinais; g) abordem 
estratégias de gamificação; h) não estejam disponíveis para acesso através dos periódicos assinados pela Universidade Federal de Santa Catarina (UFSC).

Nenhuma restrição de período de publicação foi estabelecida. Foram considerados somente publicações revisadas por pares, publicadas em espanhol, inglês e português.

\subsection{Fase 2 - Processamento}

Esta fase consiste em conduzir as buscas na base de dados, analisar os documentos em três momentos - filtros de leitura - e documentar os resultados encontrados. A análise dos documentos é feita em três momentos, com filtros baseados no RBS Roadmap (Conforto, Amaral e Silva, 2011): 1) leitura de título, resumo e palavraschave para verificar pertinência ao tema, e disponibilidade de acesso [nesta ordem]; 2) leitura da introdução e conclusão; e 3) leitura completa dos documentos.

Na busca realizada em maio de 2019 foram encontrados 397 na base de dados SCOPUS e 41 na base de dados ERIC (restritos aos documentos revisados por pares). Destes um total de 419 documentos não duplicados foram selecionados. Após o primeiro filtro - leitura do título, do resumo, das palavras-chave e disponibilidade de acesso, 99 documentos foram considerados relacionados ao objetivo da pesquisa. No segundo filtro, que consiste numa visão geral do documento, além da leitura da introdução e conclusão, 44 documentos foram selecionados para leitura completa e coleta dos dados da pesquisa. A seguir é apresentada a etapa de síntese e resultados, proposta para a terceira fase do RBS Roadmap.

\subsection{Fase 3 - Síntese e Resultados}

A partir da leitura completa dos documentos dividimos eles com base nos jogos apresentados por cada um. Dessa maneira a Tabela 1 apresenta: o título do jogo; quais os autores que os descrevem (em jogos apresentados em mais de uma publicação apenas a publicação mais recente foi citada); uma breve descrição do jogo; qual a mecânica do jogo; como é apresentada e utilizada a língua de sinais em cada um deles e; quais os objetivos de aprendizagem ou tema abordado pelo jogo. Tal caracterização é baseada na tétrade dos elementos básicos constituintes dos jogos proposta por Schell (2014), composta por: mecânica; estória; estética e tecnologia.

\section{Discussão dos resultados}

As publicações coletadas durante a revisão e descritas na Tabela 1 nos permitem traçar um estado da arte dos jogos educativos digitais que utilizam línguas de sinais. Pode-se perceber por exemplo, que a maioria dos jogos documentados aqui são jogos que baseiam-se em mecânicas simples como os jogos de correspondência. Dos 24 diferentes jogos descritos nos documentos, 14 são jogos de correspondência. Talvez o mais conhecido nesse estilo seja o jogo da memória, onde o usuário precisa encontrar e combinar imagens correspondentes. No caso de jogos como o MemoSign, os pares não são formados somente por imagens, mas por imagens e escrita de sinais; palavras e escritas de sinais correspondentes; e cálculos e seus resultados em escritas de sinais (Bouzid, 2016). A língua de sinais nesse caso, aparece na em sua forma escrita e em uma animação de um avatar tridimensional que é executada quando o usuário passa o mouse sobre a carta que contém a escrita de sinais. 
TABELA 1a - Documentação dos jogos descritos nas publicações

\begin{tabular}{l} 
Nome do jogo (Autores publicação mais recente) \\
\hline Descrição
\end{tabular}

Mecânica

Representação Língua Sinais

Objetivo de aprendizagem, Tema
ASL Learning (Kamnardsiri et al, 2016)

Jogo de captura de sinais com Microsoft Kinect.

Imagem é apresentada e o usuário deve realizar o sinal correspondente.

\begin{tabular}{l}
\hline Auslan for Kinder (Ellis, 2009) \\
\hline Multimídia interativa contendo: vocabulário; livro \\
de história; música sinalizada; jogo. \\
Jogo: usuário deve selecionar em uma imagem o \\
animal indicado por um sinal.
\end{tabular}

Jogo de correspondência [sinalizando]

Vídeo gravado: especialista apresenta alguns sinais em glossário

Língua de sinais

Jogo de correspondência [clicando]

Vídeo gravado: vocabulário, livro de história e interpretação de música.

Personagem 2D: faz o sinal de um animal que deve ser selecionado no jogo

Língua de sinais

Jogo de correspondência [clicando]

CD-ROM on Austrian sign language

(Hilzensauer, 2004)

Multimídia interativa contendo seções: diálogos; exercícios; jogos; gramática da LS; vídeos sobre cultura surda.

Jogo: selecionar num jogo da velha o quadrante indicado pelo intérprete.

\begin{tabular}{l|l}
\hline CopyCat (Zafrulla et al., 2011) & Jogo de correspondência [sinalizando] \\
\hline $\begin{array}{l}\text { Usuário deve sinalizar uma frase para indicar a } \\
\text { ação que o personagem do jogo deve realizar. Jogo } \\
\text { de input de sinais com luva e Kinect. }\end{array}$ & $\begin{array}{l}\text { Vídeo gravado: quando acionado, intérprete } \\
\text { demonstra o que deve ser sinalizado }\end{array}$ \\
\cline { 2 - 2 } $\begin{array}{l}\text { Crosswords Game for Deaf } \\
\text { (Chebka e Essalmi, 2015) }\end{array}$ & Jogo de correspondência [palavras cruzadas] \\
\hline $\begin{array}{l}\text { Usuário deve completar um jogo de palavras } \\
\text { cruzadas a partir de imagens e seus sinais } \\
\text { correspondentes apresentado por intérprete. }\end{array}$ & Vídeo gravado: sinaliza o que deve ser escrito \\
\hline \hline GeePerS*Math (Shelton e Parlin, 2016) & Língua de sinais \\
\hline $\begin{array}{l}\text { Jogo mobile para aprendizado de matemática. } \\
\text { Baseado numa narrativa perpassada por algumas } \\
\text { questões matemáticas. }\end{array}$ & $\begin{array}{l}\text { Avatar 3D: pode ser habilitado/desabilitado para } \\
\text { sinalizar as instruções e narrativa do jogo }\end{array}$ \\
\hline \hline GyGSLA (Sousa et al., 2016) & Matemática \\
\hline $\begin{array}{l}\text { Usuário deve repetir um sinal apresentado em } \\
\text { texto, em desenho e por um avatar. O sistema } \\
\text { reconhece através de luva. Jogo de input de sinais. }\end{array}$ & Jogo de correspondência \\
\hline \hline Kinect-Sign & Avatar 3D e desenho de configuração de mão: \\
dGameiro, Cardoso e Rybarczyk, 2013) & Quizz de sinais \\
\hline $\begin{array}{l}\text { Sistema de captura de sinais com Microsoft Kinect. } \\
\text { Quizz: usuário deve sinalizar de acordo com a } \\
\text { pergunta em texto. }\end{array}$ & $\begin{array}{l}\text { Desenho de configuração de mão: apresenta os } \\
\text { sinais das possíveis respostas do quizz }\end{array}$ \\
\hline \hline $\begin{array}{l}\text { LMMGDL Learning version of Memory Match } \\
\text { Game for Deaf Learner (Khenissi, 2015) Jogo da }\end{array}$ & Língua de sinais \\
\hline $\begin{array}{l}\text { memória com pares: palavras - Sign Writing (SW). } \\
\text { O avatar 3D apresenta gestualmente o sinal notado } \\
\text { em SW ao usuário clicar na carta }\end{array}$ & Avatar 3D e SW \\
\hline \hline
\end{tabular}

Vídeo gravado: intérprete sinaliza uma direção que deve ser selecionada no jogo

Língua de sinais 
TABELA 1b - Documentação dos jogos descritos nas publicações

\begin{tabular}{l} 
Nome do jogo (Autores publicação mais recente) \\
\hline Descrição
\end{tabular}

MathSigner TM

(Adamo-Villani, Brisbin e Hyland, 2006)

Questões matemáticas devem ser respondidas em

LS - captura movimento - ou através do teclado.
Mecânica

Representação Língua Sinais

Objetivo de aprendizagem, Tema

\begin{tabular}{l}
\hline MatLIBRAS Racing \\
(Pontes, Duarte e Pinheiro, 2018) \\
\hline Usuários devem selecionar respostas às operações \\
matemáticas para mover seu carro competidor.
\end{tabular}

Memosign - Derivado do LMMGDL

(Bouzid, 2016)

Jogo da memória, pares: imagem - SW; palavra -

$\mathrm{SW}$; e cálculo - SW (resultado)

Operações matemáticas

Avatar 3D: apresenta o resultado da operação

matemática em LS

Matemática

Operações matemáticas

Desenho de configuração de mão de números Matemática; Língua de Sinais

Jogo de correspondência [jogo da memória]

Operações matemáticas

Avatar 3D e SW: avatar 3D apresenta gestualmente

o sinal notado em SW ao usuário clicar na carta

Língua de sinais; SW e operações matemáticas

Jogo de correspondência

(Bento, Cláudio e Urbano, 2014)

Dicionário interativo com modo jogo. Usuário precisa escolher, dentre uma série de sinais, qual diz respeito à palavra apresentada.

Avatar 3D: apresenta uma série de sinais para relacionar com uma palavra apresentada Língua de sinais

Salabil (Silva, Franco e Okuyama, 2018)

Plataforma para ensino de surdos que pode ser adaptado para ensino de sinais em qualquer temática.

\begin{tabular}{l}
\hline Sign Language Tutor \\
(Ackovska e Kostoska, 2014) \\
\hline Aplicação com 3 módulos: glossário com avatar \\
3D animado; Jogo de memória (sinal - letra); Jogo \\
2D de plataforma (recompensas de objetos \\
coletados são animações 3D de sinais)
\end{tabular}

Jogo de correspondência [quizz e memória]

Sinal desenhado e SW

Língua de sinais (qualquer temática)

Jogo de correspondência [jogo da memória]

Jogo de exploração [plataforma 2D]

Avatar 3D no glossário;

Desenho do sinal no jogo da memória;

Animação 3D de sinal como recompensa da coleta

de itens no jogo de exploração.

Língua de sinais

Sign my world (Korte, Potter e Nielsen, 2014)

Jogo de apontar e clicar

Ambiente interativo de apontar e clicar. Ao clicar nos objetos a palavra em inglês e o sinal que o designam são exibidos.

\begin{tabular}{l|l}
\hline \hline SMILE (Adamo-Villani e Wright, 2007) & Jogo de exploração [mundo virtual 3D] \\
\hline $\begin{array}{l}\text { Jogo imersivo de mundo virtual explorável com } \\
\begin{array}{l}\text { avatares sinalizantes. Problemas de matemática e } \\
\text { ciências para solucionar. }\end{array}\end{array}$ & $\begin{array}{l}\text { Avatares 3D: personagens que interagem em LS } \\
\text { com os usuários }\end{array}$ \\
\hline \hline Terra Libras (Ferreira et al., 2016) & $\begin{array}{l}\text { Língua de sinais; Ciências; Matemática } \\
\text { Jogo da forca }\end{array}$ \\
\hline $\begin{array}{l}\text { Pacote com dois jogos: jogo da memória com o par } \\
\text { sinal - letra; e jogo da forca, palavra deve ser } \\
\text { montada com sinal e letras. }\end{array}$ & Desenho de configuração de mão - alfabeto \\
\cline { 2 - 2 } Virtual Sign (Escudeiro et al., 2017) & Língua de sinais \\
\hline \hline $\begin{array}{l}\text { Jogo de exploração num ambiente virtual. Para } \\
\text { poder coletar objetos usuário deve sinalizar para o } \\
\text { jogo - captura com Microsoft Kinect e Luvas 5DT }\end{array}$ & Exploração mundo virtual \\
\cline { 2 - 2 } & Língua de Sinais \\
\hline \hline
\end{tabular}


TABELA 1c - Documentação dos jogos descritos nas publicações

\begin{tabular}{|c|c|}
\hline Nome do jogo (Autores publicação mais recente) & Mecânica \\
\hline \multirow[t]{2}{*}{ Descrição } & Representação Língua Sinais \\
\hline & Objetivo de aprendizagem, Tema \\
\hline S/N \#1 (Krastev et al., 2014) & Jogo de Correspondência \\
\hline \multirow{2}{*}{$\begin{array}{l}\text { Usuário deve escolher a mão que sinaliza o valor } \\
\text { correspondente ao apresentado textualmente. }\end{array}$} & Imagem 3D estática de mão robótica \\
\hline & Língua de Sinais, Matemática \\
\hline S/N \#2 (Moustakas et al., 2011) & Caça ao tesouro \\
\hline \multirow{2}{*}{$\begin{array}{l}\text { Jogo de caça ao tesouro para comunicação entre } \\
\text { surdos e cegos, com captura de sinais e adaptação } \\
\text { de estímulos (auditivo - háptico - visual) }\end{array}$} & $\begin{array}{l}\text { Avatar 3D sinaliza mensagem auditiva enviada } \\
\text { pelo usuário cego }\end{array}$ \\
\hline & - \\
\hline S/N \#3 (Eqab e Shanableh, 2017) & Jogo de correspondência [quizz] \\
\hline \multirow{2}{*}{$\begin{array}{l}\text { Aplicativo com } 4 \text { funcionalidades - captura de SL } \\
\text { para treino; para tradução; voz para SL e; jogo: } \\
\text { usuário deve responder qual texto corresponde ao } \\
\text { sinal exibido pela animação. }\end{array}$} & Desenho de sinais animados (apenas mão) \\
\hline & Língua de sinais \\
\hline S/N \#4 (Nakpong e Chanchalor, 2019) & Jogos de resolução de problemas \\
\hline \multirow[t]{2}{*}{$\begin{array}{l}\text { Multimídia com } 6 \text { mini jogos com problemas } \\
\text { distintos para aprimorar inteligência emocional }\end{array}$} & $\begin{array}{l}\text { Vídeo gravado: intérprete descreve o jogo; } \\
\text { intérprete provoca reflexão sobre tema do jogo }\end{array}$ \\
\hline & Inteligência emocional e social \\
\hline S/N \#5 (Canteri et al., 2015) & Quebra-cabeça \\
\hline \multirow{2}{*}{$\begin{array}{l}\text { Jogo de montar quebra-cabeça. É preciso encaixar } \\
\text { imagem - sinal - palavra. }\end{array}$} & Desenho de sinais: peças do quebra-cabeça \\
\hline & Língua de sinais \\
\hline
\end{tabular}

Conforme Bouzid, o uso de personagens humanos tridimensionais em jogos para crianças surdas é recorrente (2016). Isso pode ser observado também aqui, pois 10 dos 24 jogos descritos apresentam avatares tridimensionais em suas interfaces em algum momento. Contudo, nesses exemplos - de jogos de correspondência - as potencialidades de representação das mídias digitais - como as animações tridimensionais - muitas vezes ficam restritas as exibições de vocabulário.

Já jogos como VirtualSign (Escudeiro et al., 2017) e SMILE (Adamo-Villani e Wright, 2007), por estruturarem a experiência do jogo em mundos virtuais tridimensionais passíveis de exploração, aproveitam melhor tais características de representação. Além disso, ambos os jogos utilizam tecnologias de input de sinais a partir de dispositivos de captura visual ou cinética, como luvas ou câmeras.

O emprego de tecnologias de captura de movimento é recorrente entre os estudos coletados. Alguns jogos apresentados foram desenvolvidos com objetivo de testar e avaliar tal recurso, dando mais ênfase ao reconhecimento correto de sinais, sua interação com o sistema do jogo e representação na interface gráfica. É o caso dos jogos Kinect-sign (Gameiro, Cardoso e Rybarczyk, 2013) e ASL Learning (Kamnardsiri et al., 2016), que utilizam o dispositivo Microsoft Kinect. Já CopyCat que é descrito em oito publicações, em suas mais recentes versões (Zafrulla et al., 2011) foi adaptado para uso em conjunto com Kinect e uma luva com acelerômetro, já descrita nos estudos anteriores deste mesmo jogo. A combinação de dispositivos de captura luva e Kinect também foi utilizado no VirtualSign (Escudeiro et al., 2017). Em SMILE foram utilizadas duas formas distintas de captura de movimento para interação com o sistema do jogo: luvas de rastreamento e bastões de captura. Ambos usados em conjunto com sistemas imersivos de realidade virtual (Adamo-Villani e Wright, 2007)

SMILE, assim como GeePerS*Math (Shelton e Parlin, 2016), Mathsigner (AdamoVillani; Brisbin e Hyland, 2006), MatLIBRAS Racing (Pontes, Duarte e Pinheiro, 2018) 
e parte do MemoSign (Bouzid, 2016) têm outra característica que os diferenciam dos demais: são jogos para aprendizado de conceitos de matemática e ciência além da aquisição da linguagem. Os demais estão restritos à aquisição de linguagem, baseandose, para tanto, em atividades nas quais o usuário deve encontrar elementos correspondentes na interface, geralmente baseados nas combinações imagem-sinal ou palavra-sinal. A única exceção é o jogo descrito por Nakpong e Chanchalor (2019) (S/N \#4 da Tabela 1c), que tem por objetivo aprimorar a inteligência social e emocional de crianças surdas de 13 a 15 anos através de jogos de situação problema que desafiam os sujeitos a refletirem sobre os temas do jogo.

Outra questão que fica evidente ao relacionar os jogos documentados é a falta de jogos em que a LS seja utilizada para comunicar elementos da narrativa ou história do jogo. Esse uso foi documentado em dois aplicativos: no CD-ROM on Austrian sign language (Hilzensauer, 2004) e o Auslan for Kinder (Ellis, 2009), no módulo de contação de histórias do aplicativo, ou seja, não durante o jogo; e no GeePerS*Math (Shelton e Parlin, 2016), em que o usuário interage com uma sequência narrativa intercalada por desafios matemáticos. No primeiro, CD-ROM on Austrian [...] a história é contada através de um intérprete filmado sobre fundo azul. Outros 7 jogos documentados utilizam vídeos gravados de intérpretes durante a interação - como forma de instrução, de ajuda etc - como o ASL Learning (Kamnardsiri, et al, 2016), o Auslan for Kinder (Ellis, 2009) entre outros. No GeePerS*Math a narrativa é apresentada em inglês escrito com alternativa de habilitar a exibição de um avatar tridimensional sinalizando o conteúdo. $\mathrm{O}$ uso desse recurso foi avaliado pelos proponentes do jogo.

Isso levanta outro ponto comum entre as publicações: o relato de avaliação do uso da língua de sinais e a compreensão desta quando é transmitida a partir de um avatar tridimensional ou desenho.

$\mathrm{Na}$ avaliação do GeePerS*Math (Shelton e Parlin, 2016) por exemplo, as crianças com surdez profunda pediram que a língua de sinais fosse utilizada ao longo de todo o jogo, pois o Inglês escrito era mais difícil de compreender do que a língua de sinais.

Entre as principais observações feitas a partir da coleta dos documentos destacam-se portanto os principais resultados na Tabela 2.

TABELA 2 - Principais resultados de pesquisa

\begin{tabular}{ll}
\hline \hline - & jogos de correspondência (i.e. jogo da memória) são os formatos mais comuns, 14 entre os 24 \\
jogos descritos utilizam essa mecânica;
\end{tabular}

\section{Considerações}

O método de revisão sistemática, conforme já apontado, permite traçarmos de maneira confiável um estado da arte do tema pesquisado. Nesse caso, ao coletar documentos que abordassem o desenvolvimento de jogos digitais que utilizem línguas de sinais, foi possível obter uma visão geral do que, pelo menos no contexto da academia, produz-se de jogos digitais em LS. 
A pesquisa empreendida para fornecer dados para trabalhos futuros na área de desenvolvimento de jogos digitais educacionais não teve em seu string de busca o termo "educação". Tampouco foi utilizado como critério de inclusão o objetivo educacional do jogo. Essa estratégia foi traçada para ampliar as possibilidades de resultados. Contudo, pode-se observar que todas as publicações sem exceção documentaram o desenvolvimento ou avaliação de jogos voltados para a educação e desenvolvidos no contexto da academia. Isso aponta para uma possível escassez ou até mesmo ausência de jogos digitais comerciais que utilizem LS. Cabe portanto, uma pesquisa em outras bases de dados que não as acadêmicas para buscar tais artefatos.

Entre as contribuições dessa pesquisa para o desenvolvimento de novos jogos digitais educacionais com LS destacam-se as seguintes: aproveitamento de modelos de aplicação de dispositivos de input/captura de sinais; aproveitamento dos resultados de pesquisa quanto ao reconhecimento, formas de uso e aceitação das LS no contexto dos jogos; lacunas nos tipos e mecânicas de jogos com LS; lacunas na exploração de formas de narrativa em LS no contexto dos jogos; e lacunas na exploração de formas de representação dos sinais ao longo dos jogos.

A partir das lacunas observadas, são propostos os seguintes estudos futuros:

- projetar jogos com LS baseados em mecânicas mais diversificadas que jogos de correspondência.

- avaliar o uso da LS em jogos com narrativas mais relacionadas à mecânica do jogo. É possível fomentar a participação do surdo apenas pelo emprego da LS como principal forma de comunicação?

Por fim, conforme proposto por Conforto, Amaral e Silva (2011) na terceira fase do RBS Roadmap, utilizaremos as ferramentas de rastreamento e alerta de novos documentos disponíveis nas bases de dados para coletar novas publicações que atendam aos requisitos de busca traçados para esta pesquisa. Dessa forma é possível manter atualizada a visão do estado da arte da temática jogos digitais educacionais em línguas de sinais.

\section{Referências}

ACKOVSKA, N.; KOSTOSKA, M. Sign language tutor - Rebuilding and optimizing. 2014 37th International Convention on Information and Communication Technology, Electronics and Microelectronics, MIPRO 2014 - Proceedings. 2014

ADAMO-VILLANI, N.; BRISBIN, M.; HYLAND, B. A Natural Interface for sign language mathematics. Lecture Notes in Computer Science (including subseries Lecture Notes in Artificial Intelligence and Lecture Notes in Bioinformatics), v. 4291 LNCS, p. 70-79, 2006.

ADAMO-VILLANI, N.; WRIGHT, K. SMILE : an immersive learning game for deaf and hearing children. Acm Siggraph, p. 17, 2007.

BENTO, J.; CLÁUDIO, A. P.; URBANO, P. Avatares em língua gestual portuguesa - Avatars on Portuguese Sign Language. Iberian Conference on Information Systems and Technologies, CISTI. 2014

BOUZID, Y.; KHENISSI, M. A.; ESSALMI, F.; JEMNI, M. Using Educational Games for Sign Language Learning - A SignWriting Learning Game: Case Study. Journal of Educational Technology \& Society, v. 19, n. 1, p. 129-141, 2016. 
BUSARELLO, R. I. Gamificação em histórias em quadrinhos hipermídia: diretrizes para construção de objeto de aprendizagem acessível. Florianópolis: PPGEGC/UFSC, 2016. 352 p. Tese de Doutorado

CAMPELO, A. R. S. Pedagogia Visual / Sinal na Educação dos Surdos. In: QUADROS, R. M. e PERLIN, G. (Orgs.) Estudos Surdos II. Petrópolis: Arara Azul, 2007. p.100-131.

CANTERI, R. P.; GARCÍA, L. S.; SOUZA, T. A. F.; IATSKIU, C. A. E. Video Games in Education of Deaf Children - A Set of Pratical Design Guidelines. ICEIS 2015 17th International Conference on Enterprise Information Systems, Proceedings. 2015

CHEBKA, R.; ESSALMI, F. A crosswords game for deaf. 2015 5th International Conference on Information and Communication Technology and Accessibility, ICTA 2015.

CONFORTO, E. C.; AMARAL, D. C.; SILVA, S. L. Roteiro para revisão bibliográfica sistemática: aplicação no desenvolvimento de produtos e gerenciamento de projetos. In 8o Congresso Brasileiro de Gestão de Desenvolvimento de Produto - CBGDP 2011.

ELLIS, K. Multimedia for primary school children learning sign language. Proceedings of the 21st Annual Conference of the Australian Computer-Human Interaction Special Interest Group on Design: Open 24/7 - OZCHI '09. 2009

EQAB, A.; SHANABLEH, T. Android mobile app for real-time bilateral Arabic sign language translation using leap motion controller. 2017 International Conference on Electrical and Computing Technologies and Applications, ICECTA 2017, 2018.

ESCUDEIRO, P., ESCUDEIRO, N., NORBERTO, M., LOPES, J.; SOARES, F. Digital Assisted Communication. In Proceedings of the 13th International Conference on Web Information Systems andTechnologies (WEBIST2017), 2017, p.395-402

FERREIRA, V.; GOMES, H.; FRAGOSO, F.; QUEIROZ, M.; PALMEIRA, R.; GUIMARÃES, A.; MELO, F.; SILVA, D.. Terra Libras: Um serious game para auxiliar no aprendizado de Libras. CEUR Workshop Proceedings. 2016

GAMEIRO, J.; CARDOSO, T.; RYBARCZYK, Y. Kinect-Sign, Teaching Sign Language to "Listeners" through a Game. 9th IFIPWG 5.5 International Summer Workshop on Multimodal Interfaces, eNTERFACE 2013. 2013

HILZENSAUER, M. CD-ROM on Austrian sign language: Course OEGS 1, lesson 1-6. Lect Notes Comput Sc Lect Notes Comput Sc. 2004

JAPPUR, R. F. Modelo conceitual para criação, aplicação e avaliação de jogos educativos digitais. Florianópolis: PPGEGC/UFSC, 2014. 296 p. Tese de Doutorado

KAMNARDSIRI, T.; HONGSIT, L.; KHUWUTHYAKORN, P.; WONGTA, N. The Development of an Intelligent Game-Based System for Learning Sign Language 
with Kinect. Proceedings of the European Conference on Games-based Learning, v. 2016-Janua, p. 304-313, 2016.

KHENISSI, M. A.; BOUZID, Y.; ESSALMI, F.; JEMNI, M. A Learning game for deaf learners. Proceedings - IEEE 15th International Conference on Advanced Learning Technologies: Advanced Technologies for Supporting Open Access to Formal and Informal Learning, ICALT 2015.

KORTE, J.; POTTER, L. E.; NIELSEN, S. Great expectations: What do children expect from their technology? SIGMIS-CPR 2014 - Proceedings of the 2014 Conference on Computers and People Research. 2014

KRASTEV, A.; LEKOVA, A.; DIMITROVA, M.; CHAVDAROV, I. An Interactive Technology to Support Education of Children with Hearing Problems. Proceedings of the 15th International Conference on Computer Systems and Technologies, 2014.

MEC. Relatório sobre a Política Linguística de Educação Bilíngue - Língua Brasileira de Sinais e Língua Portuguesa. Brasília, DF: MEC/SECADI, 2014. 24p.

MOUSTAKAS, K.; TZOVARAS, D.; DYBKJAER, L.; BERNSEN, N. O.; ARAN, O. Using modality replacement to facilitate communication between visually and hearing-impaired people. IEEE Multimedia, v. 18, n. 2, p. 26-37, 2011.

PONTES, H. P., FURLAN DUARTE, J. B.; PINHEIRO, P. R. An educational game to teach numbers in Brazilian Sign Language while having fun. In Computers in Human Behavior, (January), 2018

SALEN, K.; ZIMMERMAN, E. Regras do Jogo - fundamentos do design de jogos principais conceitos - volume 1. São Paulo: Blucher, 2012.

SCHELL, J. The art of game design: a book of lenses: second edition. USA: CRC Press Taylor \& Francis Group, 2015.

SHELTON, B. E.; PARLIN, M. A. Teaching Math to Deaf/Hard-of-Hearing (DHH) Children Using Mobile Games: Outcomes with student and teacher perspectives. International Journal of Mobile and Blended Learning, v. 8, n. 1, p. 1-17, 2016.

SILVA, C. A.; FRANCO, M. H. I.; OKUYAMA, F. Y. Salabil bilingual education platform. In SIIE 2018 - 2018 International Symposium on Computers in Education, Proceedings. 2018

SOUSA, L.; RODRIGUES, J. M. F.; MONTEIRO, J.; CARDOSO, P. J. S.; LAM, R. GyGSLA: A portable glove system for learning sign language alphabet. Lecture Notes in Computer Science (including subseries Lecture Notes in Artificial Intelligence and Lecture Notes in Bioinformatics). 2016

ZAFRULlA, Z., BRASHEAR, H.; STARNER, T.; HAMILTON, H.; PRESTI, P. American sign language recognition with the kinect. Proceedings of the 13th international conference on multimodal interfaces, p. 279-286, 2011. 